\title{
The secrets of the green and white cards
}

\author{
D Hagemeister, BA, DA (SA), DipHIVMan, PGDipIRE, MPH, EMMB, FAfAllgMed, MD
}

Corresponding author: D Hagemeister (hagemeisterdt@ufs.ac.za)

Dr Dirk Hagemeister is a senior lecturer in the Department of Family Medicine, Faculty of Health Sciences, University of the Free State, Bloemfontein, South Africa

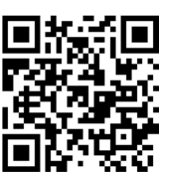

It has been more than ten years now that we have been rolling out antiretrovirals (ARVs) to the general population. And we have achieved a lot. We have successfully initiated the world's largest ARV treatment programme in South Africa, and we are starting to see the positive impact of these efforts in indicators such as life expectancy and maternal deaths.

However, there is still much room for improvement. And I am not going to talk about the sometimes erratic drug supply in our system, nor about the fact that far too many patients still arrive at the hospitals' casualty departments with advanced disease, nor about the chronic deficits with regards to resources, including human resources. However, the missing improvement that many people do not consider is the lack of adjustment in our own practice of medicine. Have we, ourselves, as clinicians in 21st century South Africa, arrived at a point where we can sufficiently meet our patients' health needs? Have we, as senior clinicians and academic teachers, internalised the need for 'clinical literacy in HIV medicine' as a basic skill that we need to teach our young colleagues at medical schools and internship sites?

Yes, maybe sometimes we have. But far too often we are not meeting these needs. In my experience teaching clinical medicine, I routinely attempt to use the learning opportunity of ward rounds to sensitise junior colleagues to the value of the simple patient-held progress cards which are routinely used in public sector antiretroviral therapy (ART) and tuberculosis (TB) services across the country. With a number of such tools being utilised in our country by now, it is striking to see the differences in familiarity with such tools between our healthcare programmes. Clinicians are fairly comfortable with extracting the relevant information from antenatal booklets and from children's 'Road-to-Health-Cards', but the same is not true for the 'green' and 'white' cards that we are using in ART and TB services. Just in case you are not terribly familiar with the two patient-held treatment cards used in the public sector for TB and HIV patients, the 'white card' is the ART progress card, usually folded into one-third of an A4 size (and often further by the patient), while the 'green card' is the A5-sized two-paged TB treatment card.

Rather saddening, the same procedure repeats itself over and over again. During ward rounds, no matter whether in casualty, adult or maternity wards, a young eager colleague presents a patient as 'known HIV and TB patient, on treatment, now admitted because of shortness of breath, diarrhoea, abdominal pains, etc.. The standard conversation then continues as such:

Me: 'When was the patient started on treatment?'

Colleague: 'I don't know.'

Me: 'Did you look at the treatment cards?'

Colleague: 'The patient didn't bring them.'

$\mathrm{Me}$, turning to the patient: 'Can you please give me the clinic card', when the patient usually grabs a plastic bag that is lying somewhere on his or her bed, containing some food supplies, money, possibly a handkerchief and the green/white cards.

What upsets me most about this exchange is not the 'taking chances' of our young colleagues - as a father of three I am used to such approaches, and after a busy night in the emergency department with multiple admissions, one probably has every reason to try to cut some corners. What really makes me sad is that we do not recognise the intrinsic value of these patientheld tools for our assessment and further management of the patient in front of us.

\section{'Have we, as senior clinicians and academic teachers, internalised the need for "clinical literacy in HIV medicine" as a basic skill that we need to teach our young colleagues at medical schools and internship sites?'}

More often than not, the TB and ART cards reveal scarring gaps in the clinical practice of these basic programmes. Patients are started on TB medication 'on clinical grounds', no sputum smear result is documented, or the intensive (four-drug) phase is ended and the continuation (two-drug) phase of pulmonary TB treatment is commenced without having confirmed sputum conversion in smear-positive cases. Similarly, HIV patients have been on 'failing regimens' for years, high viral loads were documented one to two years ago, but never followed up; worse even, single-drug substitutions have been performed in the presence of large, detectable viral loads.

Please do not get me wrong, I am not blaming the staff in our primary healthcare clinics for this - it is enough that they are often left alone in challenging supply chain settings, have no 
clinical support or supervision, and have overwhelming expectations placed on them. As far as I am concerned, we need to pull up our socks as senior clinicians and academics. Yes, HIV treatment is today where TB treatment was a couple of years ago: it is largely governed by national protocols, has been handed over to primary healthcare clinics and is done by dedicated nursing staff in those facilities.

\section{‘... we may not bother anymore to understand the basics of these programmatic treatments, as they may be perceived to be below our scope of practice. However, this attitude is going to cost us dearly ...}

But as with TB, the 'handing-over' of HIV treatment to primary healthcare services bears a real risk in clinical practice: we as medical doctors may not bother anymore to understand the basics of these programmatic treatments, as they may be perceived to be below our scope of practice. However, this attitude is going to cost us dearly as we sit on a national time-bomb of ever-increasing drug resistance in $\mathrm{TB}$, and possibly soon in HIV, too. Nurse-initiation and management of ART is an essential, and incredibly valuable step in the broad rollout of ARVs. However, it must not result in us as medical professionals 'disempowering' ourselves in terms of clinical skills in treating these conditions.

In an attempt to address this growing trend, we recently reverted to the ultimate means an academic teacher has available to emphasise the importance of a topic we teach: including the interpretation of patient-held treatment progress cards into both the postgraduate and undergraduate examinations in Family Medicine. Inevitably, the results of the examinations were rather sobering this year. But now that it is no secret anymore that green cards and white cards may be in next year's examination ...

S Afr J HIV Med 2014;15(1):33-34. DOI:10.7196/SAJHIVMED.1036 